\title{
SPECIAL ARTICLE OPEN Perspectives from the Society for Pediatric Research: Probiotic use in urinary tract infections, atopic dermatitis, and antibiotic-associated diarrhea: an overview
}

\author{
Catherine S. Forster ${ }^{1}$, Michael H. Hsieh ${ }^{1}$ and Michael D. Cabana ${ }^{2}$ \\ Probiotics have received significant attention within both the scientific and lay communities for their potential health-promoting \\ properties, including the treatment or prevention of various conditions in children. In this article, we review the published data on \\ use of specific probiotic strains for three common pediatric conditions: the prevention of urinary tract infections and antibiotic- \\ associated diarrhea and the treatment of atopic dermatitis. Research into the utility of specific probiotic strains is of varying quality, \\ and data are often derived from small studies and case series. We discuss the scientific merit of these studies, their overall findings \\ regarding the utility of probiotics for these indications, issues in reporting of methods, and results from these clinical trials, as well \\ as future areas of investigation.
}

Pediatric Research (2021) 90:315-327; https://doi.org/10.1038/s41390-020-01298-1

\section{IMPACT:}

- Review of data for commonly encountered diagnoses in the general pediatric population.

- Highlights gaps in the evidence and provides insight into new avenues of research.

\section{INTRODUCTION}

A growing understanding of the microbiome has led to a broader understanding of both health and disease. The advent of affordable advances in technology has made the ability to identify bacteria on the basis of $16 \mathrm{~S}$ rRNA sequencing feasible, leading to the discovery of the populations of bacteria both on and within the body. ${ }^{1-3}$ This prompted the Human Microbiome Project, the goal of which was to study the role of these various microbial communities. ${ }^{4}$ Much of the early work of the Human Microbiome Project focused on documenting the components of site-specific microbiomes during a normal, healthy state. More recently, investigators have begun to explore correlations between perturbations in the microbiome (e.g., dysbiosis) with specific diseases and determine the therapeutic potential of administration of exogenous bacteria or yeast (e.g., probiotics) in various conditions.

In an effort to standardize the definition of probiotics, a 2014 consensus panel recommended the following amended definition: "Probiotics are live organisms that, when given in adequate amounts, confer a health benefit to the host." ${ }^{\prime 5}$ While this concept has gained recent popularity, it was first described in the early twentieth century by Elie Metchnikoff, who hypothesized that live bacterial cultures found within yogurt had health-promoting benefits. ${ }^{6}$ Although quiescent for more than half a century, the notion of a therapeutic role of probiotics has gained relatively recent traction, with the probiotics market estimated to have exceeded $\$ 35$ billion in 2015 . $^{7}$ The field of probiotics research is still growing and is rife with studies that include small numbers of patients, use different probiotic strains, and have mixed results regarding the utility of probiotics. Further, the concept of strain specificity, in which effects of the probiotic in question are attributable to a specific strain that are not necessarily present in other strains of the same bacteria or yeast, has caused further complexity in this area of research given the lack of strain data present in early work on probiotics. ${ }^{8}$

The purpose of this review is to examine the literature on the use of probiotics in children for three common, general pediatric conditions (prevention of urinary tract infections (UTI), treatment of atopic dermatitis (AD), and prevention of antibioticassociated diarrhea (AAD)) and to highlight future areas of research.

\section{METHODS}

We conducted a scoping review to identify peer-reviewed publications of randomized controlled trials (RCTs) focused on the prevention of UTIs and AAD and the treatment of AD. We searched PubMed and Scopus from January 1, 1994 to August 1, 2020. The search included the terms "probiotics," "diarrhea," "atopic dermatitis," "urinary tract infections," and "child." All

\footnotetext{
'Department of Pediatrics, Children's National Health System, Washington, DC, USA and ${ }^{2}$ Department of Pediatrics, Children's Hospital at Montefiore and the Albert Einstein School of Medicine, Bronx, NY, USA

Correspondence: Catherine S. Forster (forstercs@upmc.edu)
}

Received: 16 April 2019 Revised: 3 November 2020 Accepted: 9 November 2020

Published online: 7 December 2020 
terms were searched as both keywords and Medical Subject Heading (MeSH) terms in PubMed (National Library of Medicine; Bethesda, MD) and as keywords in Scopus (Elsevier; Philadelphia, PA).

We included peer-reviewed publications of RCTs that focused on a pediatric population $>3$ years of age. If the range of included children overlapped with 3 years, we used the mean age of patients to determine inclusion such that trials where the mean age of the population was $>3$ years were included. This age was specifically chosen to exclude studies that focused exclusively on neonates, infants, or pregnant women. We excluded studies if the primary outcome was neither incidence or severity of $A A D$, clinical improvement in $A D$, or incidence of UTI; if the outcome was not reported in terms of clinical change; if the intervention was a probiotic product without a clearly specified probiotic organism (e.g., yogurt); if the intervention was a synbiotic or prebiotic; or if the outcome was not relevant to the general pediatrics population. For AAD studies, we excluded those that focused exclusively on an outcome of Clostridium difficile colitis. Studies that used yogurt as an intervention but specified both the probiotic strains and amounts of each probiotic within the yogurt were included.

\section{RESULTS}

The search resulted in 354 articles in PubMed and an additional 78 citations (after exclusion of duplicates) from Scopus. Three hundred and sixty six of these articles were excluded, leaving 74 for full-text review. We reviewed those 74 articles, 50 of which were excluded at the full-text review stage. The remainder were those that addressed the population, intervention, and outcomes of interest in this review (Fig. 1).

\section{Urinary tract infections}

There are limited pediatric studies focused on UTI prevention in children. We found only two RCTs on this topic. Each study used a different probiotic intervention. In both studies, the children were at risk for UTI based on vesicoureteral reflux or a history of UTI.

One study examined the utility of a combination of Lactobacillus acidophilus (LA5) and Bifidobacterium lactis (BB12) and antibiotics versus antibiotics alone. In this trial, 106 children with primary persistent vesicoureteral reflux were randomized to either prophylaxis with probiotics and nitrofurantoin or nitrofurantoin alone. There was no significant difference in the incidence of recurrent UTI between groups, but there was a lower incidence of febrile UTIs in year 3 in the probiotics group. This study, however, was limited by use of a positive urine culture as the definition of UTI, which may bias the results given that a positive urine culture does not always reflect a UTI. ${ }^{9}$ More recently, a randomized double-blind placebo-controlled trial of 244 children (181 who completed the trial) with a single, uncomplicated febrile UTI were randomized to receive either a combination of multiple strains of Lactobacillus (including $L$. acidophilus and Lactobacillus rhamnosus, strains not specified)) and Bifidobacterium species (including Bifidobacterium bifidum and $B$. lactis, strains not specified) or placebo following treatment of the acute UTI. Children in the probiotic group had a significantly lower rate of UTI recurrence after 18 months compared to children in the placebo group. ${ }^{10}$

While one study did provide some evidence that a probiotic intervention may have utility for UTI prophylaxis, further work is necessary to confirm these results (Table 1).

\section{Atopic dermatitis}

We found ten studies focused on probiotic interventions for $A D$ treatment in children. It was not possible to combine the results of the studies, as each of the studies used a different probiotic intervention or treated children with varying degrees of $A D$ severity, based on the reported SCOring Atopic Dermatitis (SCORAD) severity scores, previous medications used, or other descriptors.

There are several trials that focus on the effectiveness of single probiotic strains for AD. Han et al. randomized 118 children (83 who completed the study) to 12 weeks of Lactobacillus plantarum CJLP133 or placebo. Those in the probiotic group had a significant decrease in the SCORAD index ${ }^{11}$ at 14 weeks compared to the control group. However, this difference was no longer present at 16 weeks, suggesting that any potential beneficial effect of the probiotic does not persist after the discontinuation of therapy. ${ }^{12}$

Prakoeswa et al. randomized 22 patients $<14$ years of age with mild-to-moderate $A D$ and elevated levels of total serum immunoglobulin $\mathrm{E}(\lg \mathrm{E})$ to receive 12 weeks of either L. plantarum IS10506 or placebo. They reported decreases in SCORAD in both groups, although a larger decrease in those receiving probiotics than placebo. ${ }^{13}$

In another randomized double-blind placebo controlled study ( $n=88$ enrolled and 75 who completed the study), Woo et al. assessed the effectiveness of Lactobacillus sakei KCTC10755BP for 12 weeks and found that children 2-10 years of age in the treatment group had improved SCORAD at 12 weeks compared to placebo. ${ }^{14}$ However, a potential limitation of this work is that patients in the probiotic group had lower baseline levels of both cytokines C-C chemokine motif ligand 17 (CCL17) and CCL27, both of which are correlated with SCORAD. ${ }^{14}$

There have also been several studies focusing on various combinations of species. Wang et al. conducted a double-blind, randomized-controlled trial comparing the effectiveness of Lactobacillus paracasei GMNL-133, Lactobacillus fermentum GM090, or a combination of both, compared to placebo for 3 months for 220 children ( 212 who completed the study) aged 1-18 years with AD. They found significantly reduced disease severity, based on SCORAD, in all three probiotic groups compared to placebo. ${ }^{15}$ Sistek et al. randomized 59 children ( 49 who completed all 16 weeks of the study) aged (1-10 years) with SCORAD $\geq 10$ and either a positive skin prick test or positive radioallergosorbent test to a common food or environmental allergen to receive either 12 weeks of a combination of probiotics (L. rhamnosus and B. lactis, strains not specified) or placebo. After adjusting for baseline differences in SCORAD between groups, the authors found that only food-sensitized children in the probiotic group had a significant improvement in their SCORAD. ${ }^{16}$

Navarro-Lopez et al. conducted a double-blind placebocontrolled study and randomized 50 children (47 of whom were analyzed) 4-17 years of age to receive either a combination of $B$. lactis CECT 8145, Bifidobacterium longum CECT 7347, and Lactobacillus casei CECT 9104 or placebo for a period of 12 weeks. Those assigned to the probiotics arm had significant improvements in disease severity and decreased use of topical steroids compared to the placebo arm, although no changes were seen in any serum markers. ${ }^{17}$ However, of note, all children in the study were required to consume a high-quality Mediterranean Diet, as defined by a Mediterranean Diet Quality Index score $>7,{ }^{18}$ which may limit the generalizability of this study. ${ }^{17}$

Yesilova et al. conducted a double-blind, randomized placebocontrolled study with children ( $n=40,39$ of whom completed the study) and compared the effectiveness of a combination of Bifidiobacterium bifidium, L. acidophilus, L. casei, and Lactobacillus salivarius (strains not specified) for a period of 8 weeks. While the combination of probiotics significantly reduced disease severity, the baseline SCORAD differences between the placebo and probiotic groups were much greater than the post-intervention SCORAD, which may be due to a regression to the mean phenomenon, rather than a true difference in effectiveness. ${ }^{19}$ 


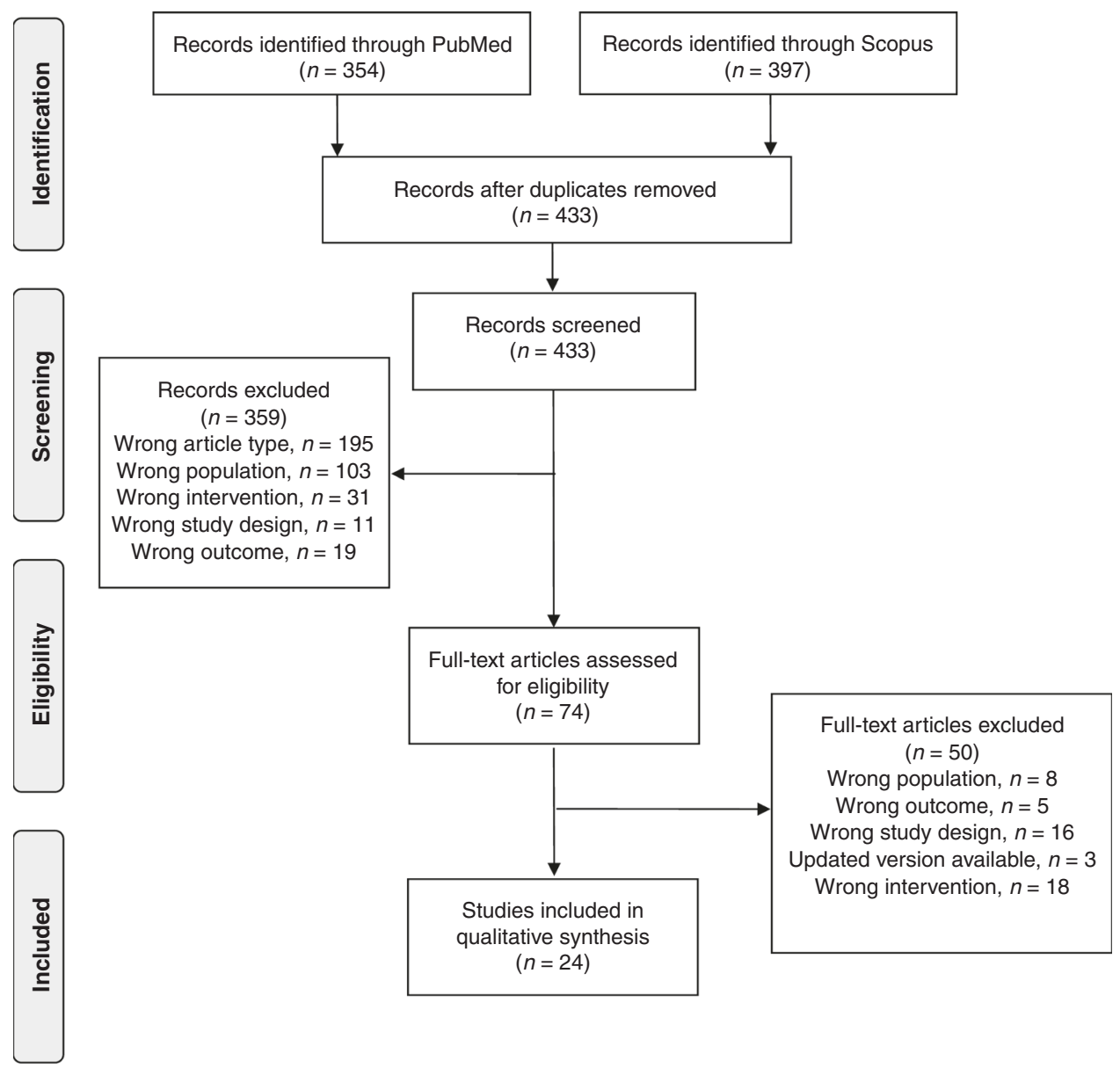

Fig. 1 PRISMA diagram of articles within this review. This figures depicts the number of articles initially identified, those exluded and their reasons, and the number of articles ulimately included in this scoping review.

Three other studies did not find a benefit to probiotic use. Yang et al. conducted a randomized study of children with mildto-moderate $\mathrm{AD}$ ( $n=100$ enrolled, 71 completed the study). They found that the combination of $L$. casei, L. rhamnosus, $L$. plantarum, and $B$. lactis (strains not specified) did not demonstrate an improvement in the probiotic group compared to the placebo. ${ }^{20}$

Rosenfeldt et al. used a crossover, placebo-controlled design and compared lyophilized L. rhamnosus 19070-2 and L. reuteri DSM 122460 to placebo. They found that patients with an allergic phenotype (defined as one positive skin prick response and elevated IgE levels) did have a significant reduction in SCORAD score during probiotic, but not placebo, treatment. Of note, the washout period was 6 weeks, which is likely sufficient in this context, although contamination and insufficient washout may confound the results. ${ }^{21}$ More recently, investigators randomized 95 children ( 82 who completed the study) to either L. pentosus (strain not specified) or placebo. While significant improvement was seen in SCORAD in both groups, there was no difference in the degree of improvement between the intervention and placebo groups ${ }^{22}$ (Table 2 ).

A recent meta-analysis reported the positive effect of probiotics on decreasing disease severity in children with AD. ${ }^{23}$ However, a recent Cochrane review noted a difference in effects based on age: while children between 2 and 12 years of age had significant improvement in SCORAD with use of probiotics, this was not seen in children under the age of 2 years. ${ }^{24}$
Antibiotic-associated diarrhea

We found 13 studies focused on probiotic interventions for the prevention of AAD. The most common probiotic interventions tested included Saccharomyces boulardii (4 studies), Lactobacillus reuteri DSM 17938 (2 studies), and Lactobacillus GG (2 studies). All the studies include children who were prescribed antibiotics in an outpatient setting and usually within $48 \mathrm{~h}$.

The majority of studies focused on single strains of probiotics, the most studied of which is $S$. boulardii. A study by Kotowska et al. randomized 269 children ( 246 of whom were analyzed) who were prescribed antibiotics for either acute otitis media or respiratory infections to either probiotic or placebo. They found a lower rates of $A A D$ in the probiotics versus placebo group (3.4 versus $17.3 \%) .{ }^{25}$ Similarly, Jindal et al. randomized 600 children given antibiotics to receive either probiotics and antibiotics or antibiotics alone. They also report a lower rate of AAD in the probiotics group compared to antibiotics alone (5.3 versus $24.0 \%$ ). Of note, this study did not include a placebo group. ${ }^{26} \mathrm{~A}$ 2013 study by Shan et al. found that patients who received antibiotics for lower respiratory tract infections and also received S. boulardii $(n=167)$ had a lower incidence of AAD than those who were not given probiotics $(n=166)$, with a relative risk of 0.22 (95\% confidence interval $(\mathrm{Cl}): 0.1-0.5)$. Further, this group also reported that initiation of $S$. boulardii in children who developed AAD but were not initially randomized to receive probiotics resulted in a decreased stool frequency compared to those in whom probiotics were not initiated. However, the 


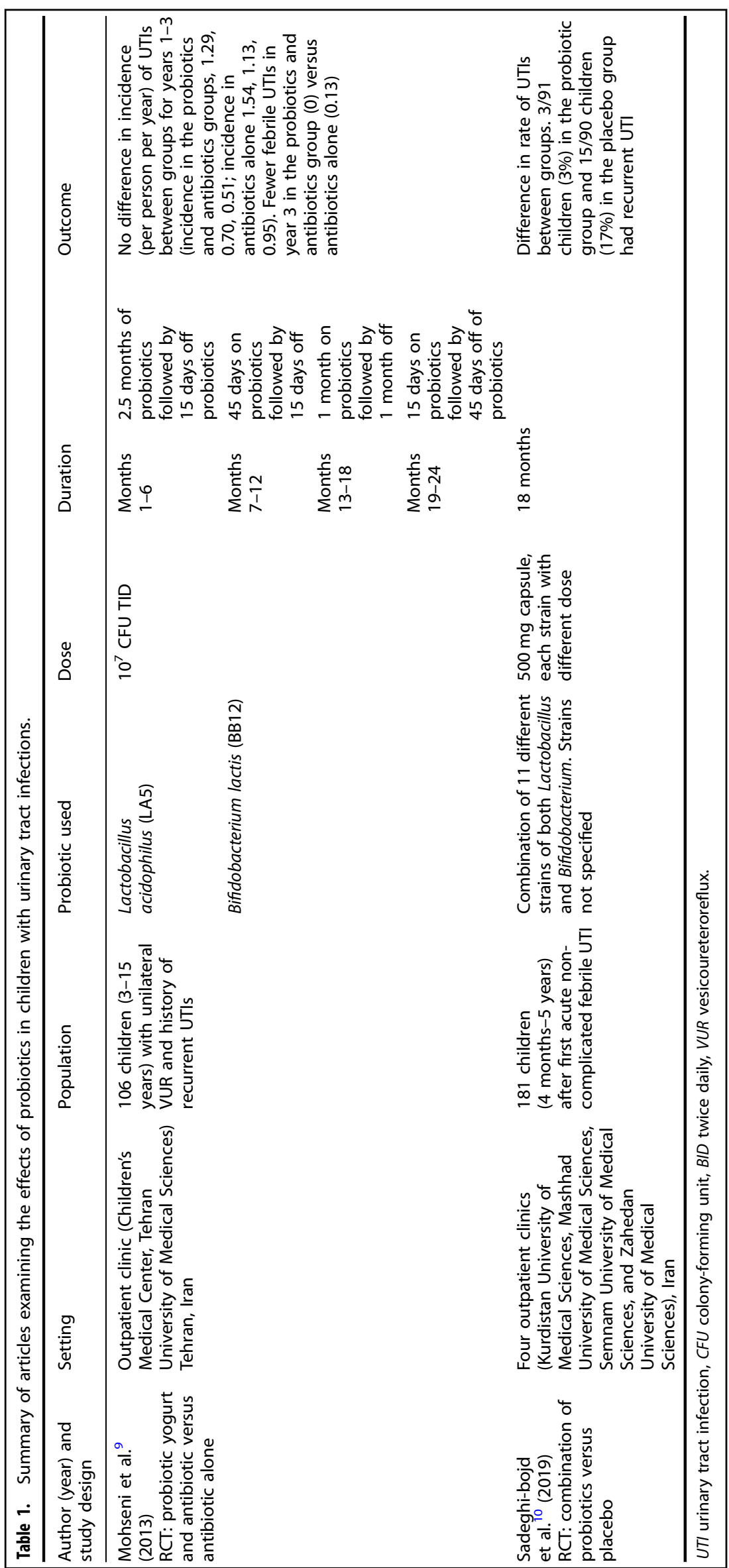




\begin{tabular}{|c|c|c|c|c|c|c|c|}
\hline & 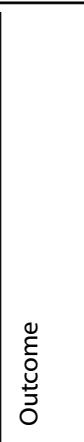 & \multicolumn{2}{|c|}{ 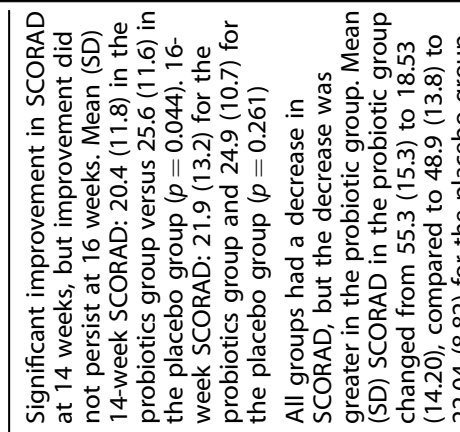 } & 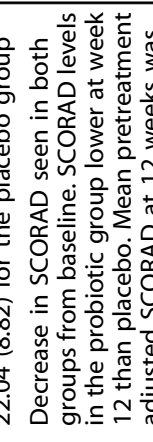 & 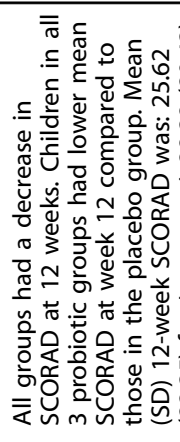 & 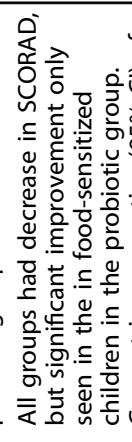 & 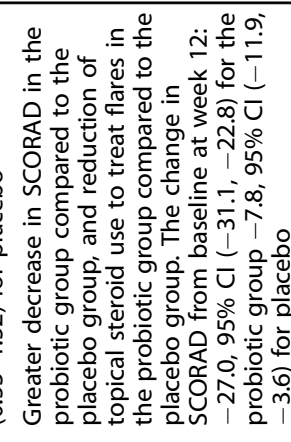 \\
\hline & 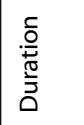 & 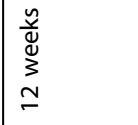 & 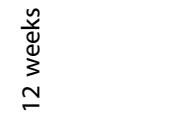 & 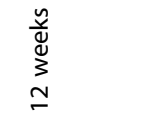 & 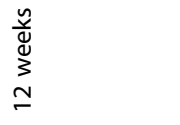 & 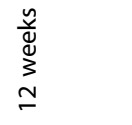 & 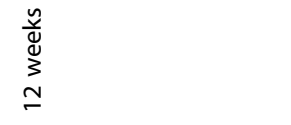 \\
\hline & Oัٌ & 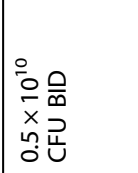 & 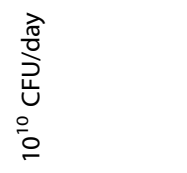 & 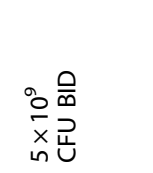 & 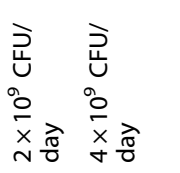 & 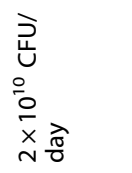 & 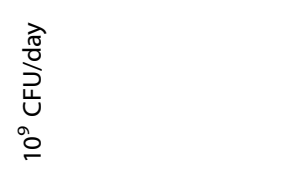 \\
\hline 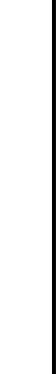 & 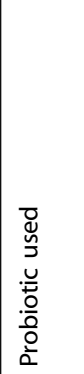 & 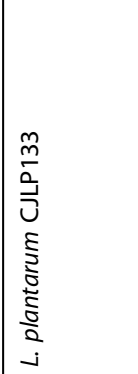 & 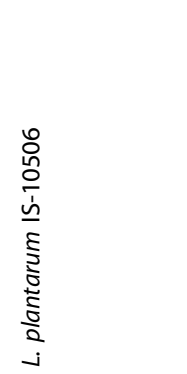 & 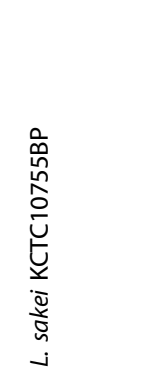 & 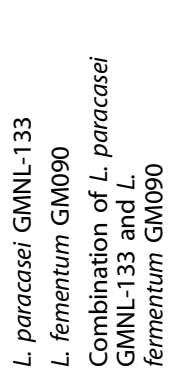 & 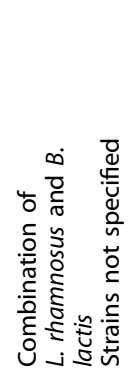 & 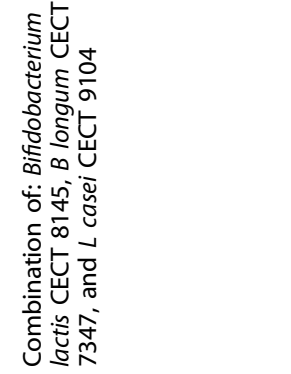 \\
\hline 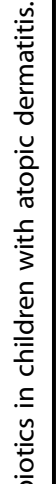 & $\begin{array}{l}\frac{1}{\circ} \\
\frac{0}{\bar{t}} \\
\frac{\pi}{\bar{z}} \\
\frac{0}{0} \\
0\end{array}$ & 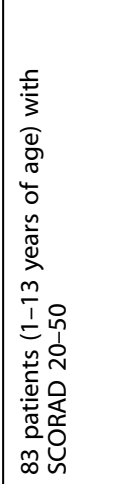 & 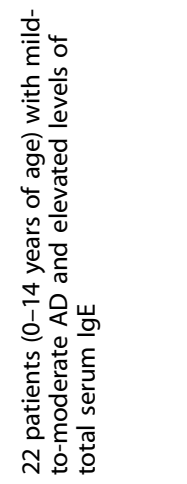 & 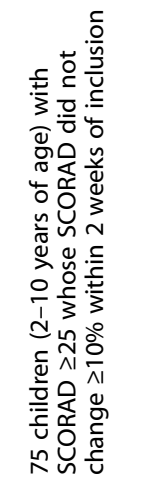 & 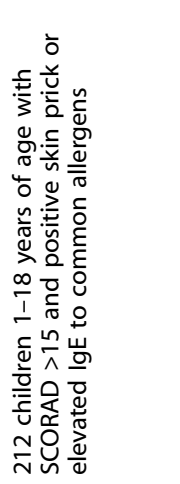 & 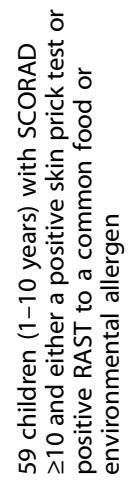 & 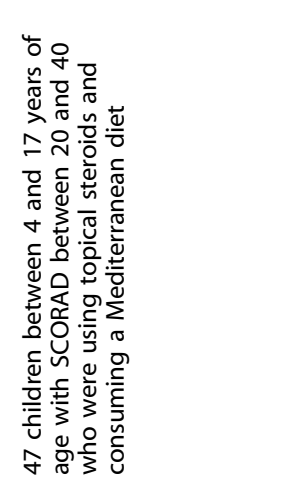 \\
\hline 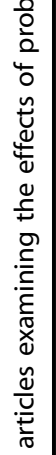 & 吕 & 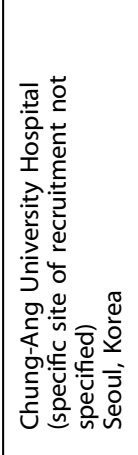 & 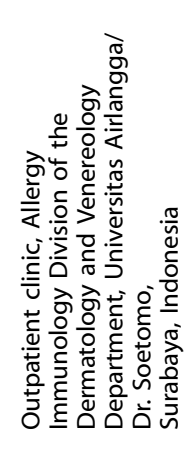 & 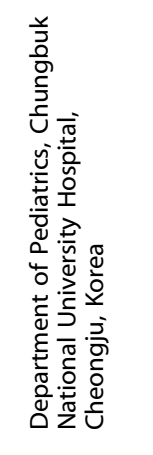 & 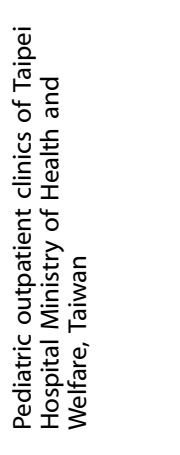 & 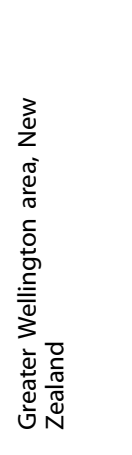 & 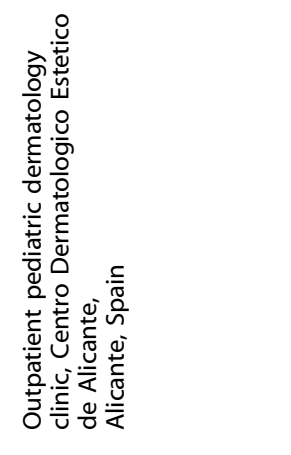 \\
\hline 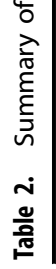 & 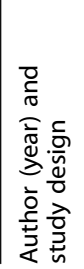 & 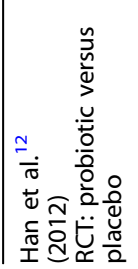 & 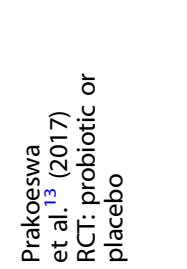 & 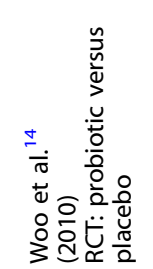 & 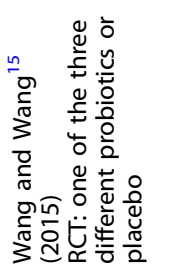 & 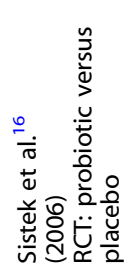 & 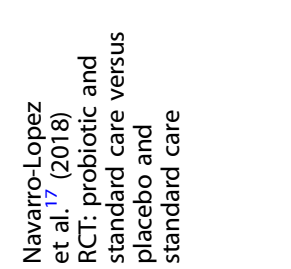 \\
\hline
\end{tabular}




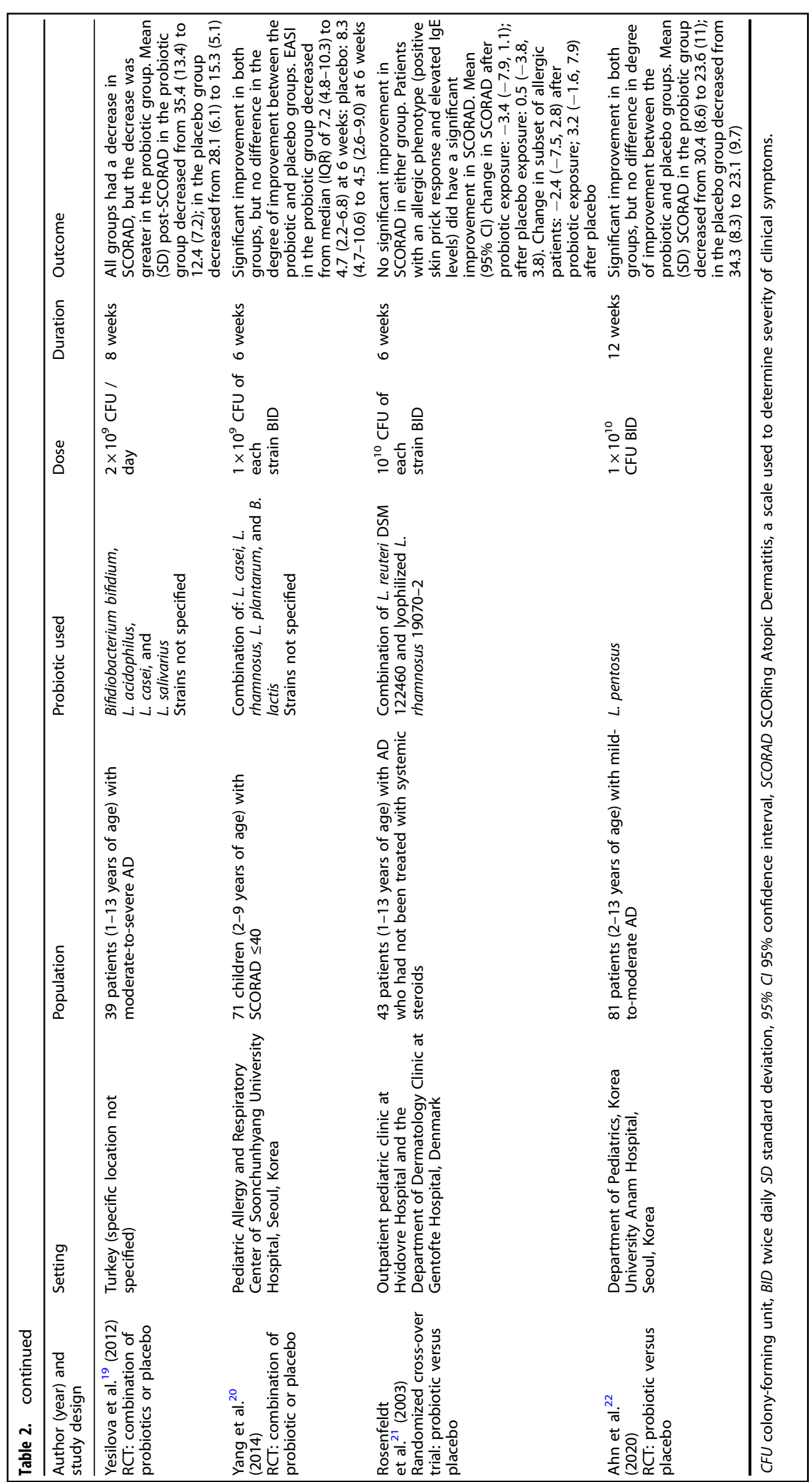


authors in this work included diarrhea due to $C$. difficile in their $A A D$ group, potentially affecting the interpretation of their results. ${ }^{27} \mathrm{~A}$ final study by Bin et al. found a lower incidence of AAD in children receiving triple antibiotic therapy for Helicobacter pylori infection in children who were given S. boulardii (CNCM 1-745) with the antibiotics compared to patients who received antibiotics alone $(11.8$ versus $28.3 \%, p<0.05) .{ }^{28}$

Other authors have focused on single strains of Lactobacillus, with mixed results. A recent multi-center study of 438 children (447 children enrolled) randomized children to either $L$. plantarum DSM9843 (LP299V)) $(n=218)$ or placebo $(n=220)$ and found that the use of probiotics did not reduce the incidence of $A A D$ or the mean number of loose, watery stools in children given antibiotics by their primary care pediatrician. ${ }^{29}$ Two studies focused on L. reuteri DSM 17938, neither of which found a difference in the incidence of AAD. ${ }^{30,31}$ However, in one of these studies, the overall rate of AAD in the entire cohort was quite low at $2 \%$, which likely contributed to their negative findings. ${ }^{30}$ Similar work has shown that Lactobacillus casei sps. rhamnosus (LGG) is beneficial, when compared to placebo, for reduction of stool frequency in children on oral antibiotics. ${ }^{32} \mathrm{~A}$ final, older study, focused on Lactobacillus casei sps. rhamnosus (Lactobacillus $G G$ ) and found that it decreased the incidence of AAD compared to placebo. ${ }^{33}$

Other studies have used combinations of strains. One study of a combination of strains of $L$. rhamnosus (strains $\mathrm{E} / \mathrm{N}, \mathrm{Oxy}$, and Pen) found that children on antibiotics for common illnesses in the probiotic $(n=120)$, as opposed to placebo $(n=120)$, group had a significant reduction of all diarrhea, defined as three or more episodes of loose and watery stools per day for $\geq 48 \mathrm{~h}$ that occurred during or up to 2 weeks following antibiotic therapy. However, there was no difference in the incidence of AAD (including diarrhea due to $C$. difficile) between the two groups. ${ }^{34}$ Another double-blind, placebo-controlled trial studied the combination of $B$. longum PL03, L. rhamnosus KL53A, and L. plantarum PL02 in 78 children exposed to antibiotics and did not find a difference in the incidence of AAD. They did note, however, a significant reduction in the number of episodes of diarrhea in the probiotic group compared to placebo. ${ }^{35}$ Another study used a different combination of probiotics that included Lactobacillus sporogenes, Streptococcus faecalis T110, Clostridium butyricum TO$\mathrm{A}$, and Bacillus mesentericus TO-A. In this work, in which the probiotic mixture was given twice a day to patients on amoxicillin-clavulanate for an unspecified amount of time, there was a decreased incidence in AAD compared to children who received antibiotics without the probiotic mixture. ${ }^{36}$ Others compared a probiotic yogurt containing Lactobacillus GG ((strain deposit number (SDN): ATCC53103), B. lactis (SDN: DSM15954), and L. acidophilus (SDN: DSM13241) with a pasteurized yogurt containing Streptococcus thermopolis and Lactobacillus bulgaricus. In this study, the authors found a decrease in both the incidence of $A A D$ and incidence of severe diarrhea in the probiotic groups. This study was limited, however, by the smaller number of patients in each group ${ }^{37}$ (Table 3 ).

We found several meta-analyses on this topic. A recent Cochrane Review, published in 2019, found an overall protective effect of probiotics and concluded that either L. rhamnosus or S. boulardii at a dose of 5-40 billion colony-forming units (CFUs)/day are the most appropriate choices for preventing AAD, with a number needed to treat to avoid one case of AAD of $9 .{ }^{38}$ Metaanalyses focused on the utility of $S$. boulardii or LGG supported these findings, with a number needed to treat of 10 for S. boulardii and 9 for $L G G{ }^{39,40}$ These strains are also recommended by the European Society for Pediatric Gastroenterology, Hepatology, and Nutrition for use in the prevention of AAD. ${ }^{41}$ Similarly, a metaanalysis on Bifidiobacterium demonstrated efficacy in the prevention of $A A D$, although the authors did note that significant heterogeneity existed in the included studies. ${ }^{42}$

\section{DISCUSSION}

In our summary of the literature on the use of probiotics for common childhood indications including UTI prophylaxis, treatment of $A D$, and prevention of $A A D$, we found varying levels of evidence.

\section{Urinary tract infections}

The dogma regarding the sterility of urine was recently challenged with the identification of the urinary microbiome. ${ }^{43-45}$ Since its initial description, a number of studies have shown decreased microbial diversity in the setting of an UTI, suggesting a potential role of the urinary microbiome in UTI pathogenesis. ${ }^{46-48}$ These studies that propose a role of the urinary microbiome in UTI pathogenesis are supported by older in vitro work. Studies suggest that Lactobacillus prevents the adherence of Gram-negative uropathogens to urothelial cells. ${ }^{49,50}$ Other work in this area demonstrated that oral administration of both $L$. rhamnosus GR-1 and L. reuteri (previously fermentum) RC-14 can colonize the vagina and decrease the presence of coliform bacteria, ${ }^{51}$ suggesting a potential role in decreasing risk of recurrent UTI. In addition, several animal models suggest that probiotic interventions may prevent UTIs. ${ }^{52,53}$

Most of the work in this area has focused on women, with little data focused on utility of probiotics in preventing UTI in children. In our analysis, we found only two RCTs regarding the use of probiotic interventions to prevent UTls in children; however, neither intervention has been replicated in other settings. For children at risk for UTIs, preventive approaches would be beneficial, given the risk of renal scarring associated with febrile UTIs.

\section{Atopic dermatitis}

The hypothesized role of the intestinal microbiome in AD occurs potentially through the interplay of the gastrointestinal (Gl) microbiota and the immune system, which can affect the development of an imbalanced immune response. While the exact mechanism is not fully elucidated, one possibility is that the intestinal microbiota can either induce immune activation or trigger immune tolerance through cytokine stimulation. ${ }^{54}$ These changes, in turn, can exacerbate the T helper type 1 (Th1)/Th2 imbalance seen in $A D$, which is associated with increased secretion of $\operatorname{lgE}{ }^{55}$ Indeed, in vitro work demonstrated that a combination of non-specific strains of $L$. acidophilus, $L$. casei, $L$. reuteri, and $B$. bifidum (strains not specified) could exert anti-inflammatory properties through induction of $\mathrm{T}$ and $\mathrm{B}$ cell hyporesponsiveness. ${ }^{56}$

Further supporting the role of the intestinal microbiome in $A D$ are several studies that have reported differences in stool microbiota between children with and without AD. ${ }^{57-59}$ Others did not find a difference in specific bacterial taxa but identified differences in functional genes related to immune development between children with and without $A D$ using metagenomic analysis of stool. ${ }^{60}$ Finally, children with $A D$ also have decreased levels of known anti-inflammatory metabolites, butyrate and propionate, in their stool. ${ }^{57}$

For children with $A D$, although there are RCTs of probiotic interventions that suggest some benefit, none of these studies have been replicated. Due to variation in the strains used, the population of interest, and the duration of treatment, comparisons are difficult. Further, several of the published works have limitations that make interpretation of their results difficult. Indeed, several studies suggest a benefit to the use of specific probiotic strains for treatment of children with $A D$; however, none of these studies have been reproduced. There is a need for large, confirmatory studies that replicate the findings of specific probiotic interventions for treatment of $A D$ in children.

\section{Antibiotic-associated diarrhea}

$A A D$ is defined as the presence of unexplained diarrhea that occurs during a course of antibiotics. One mechanism through 


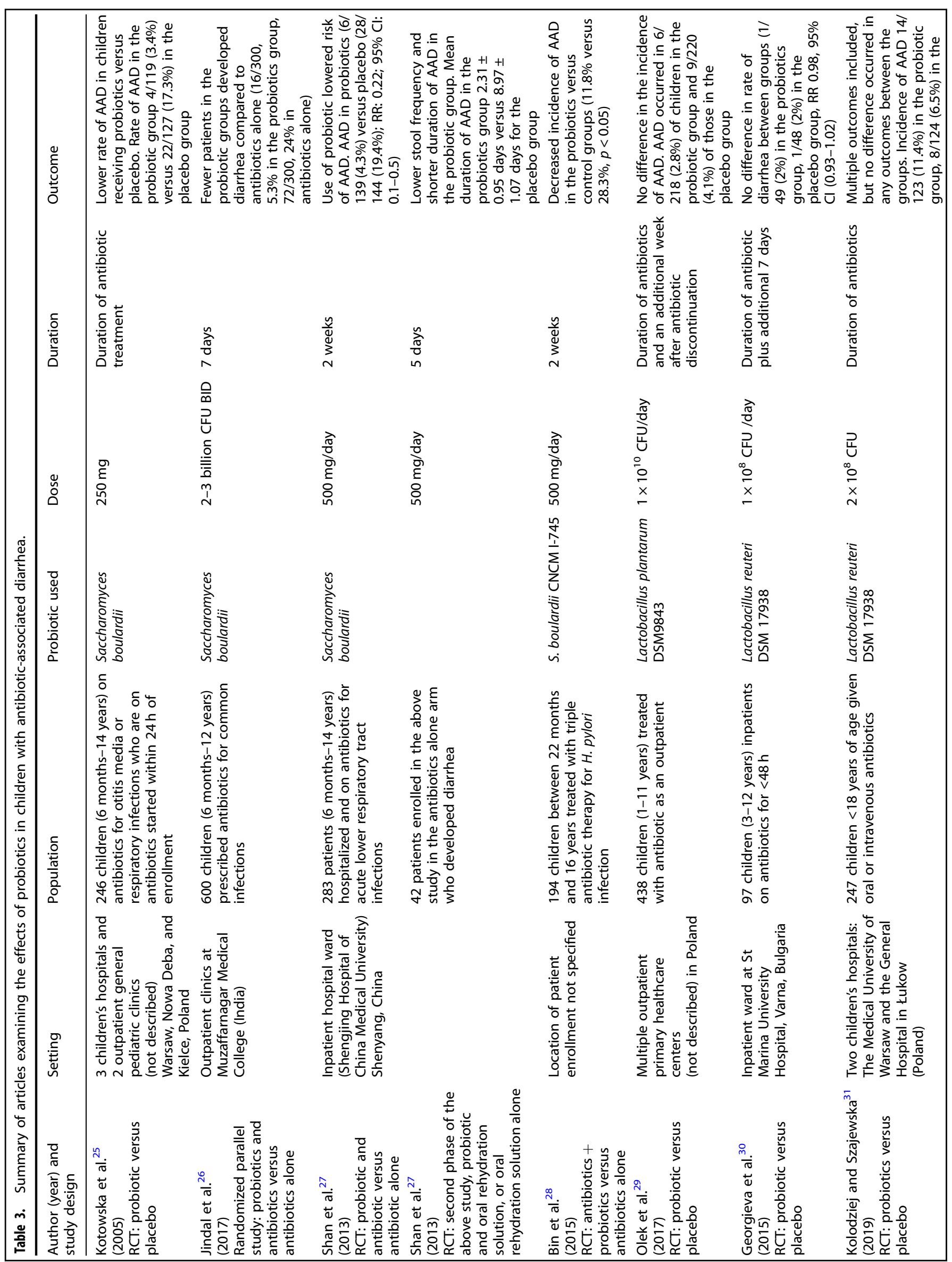




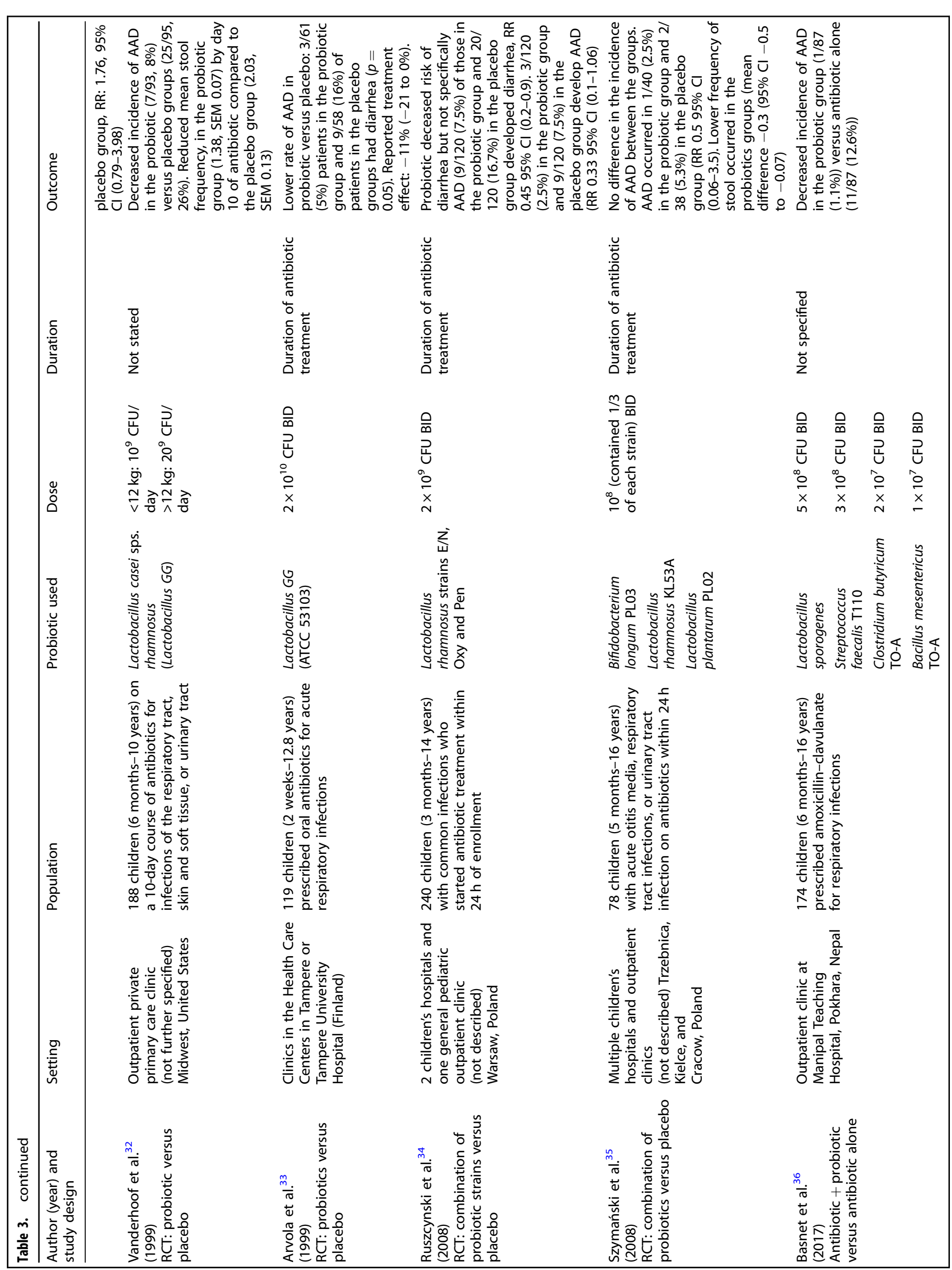




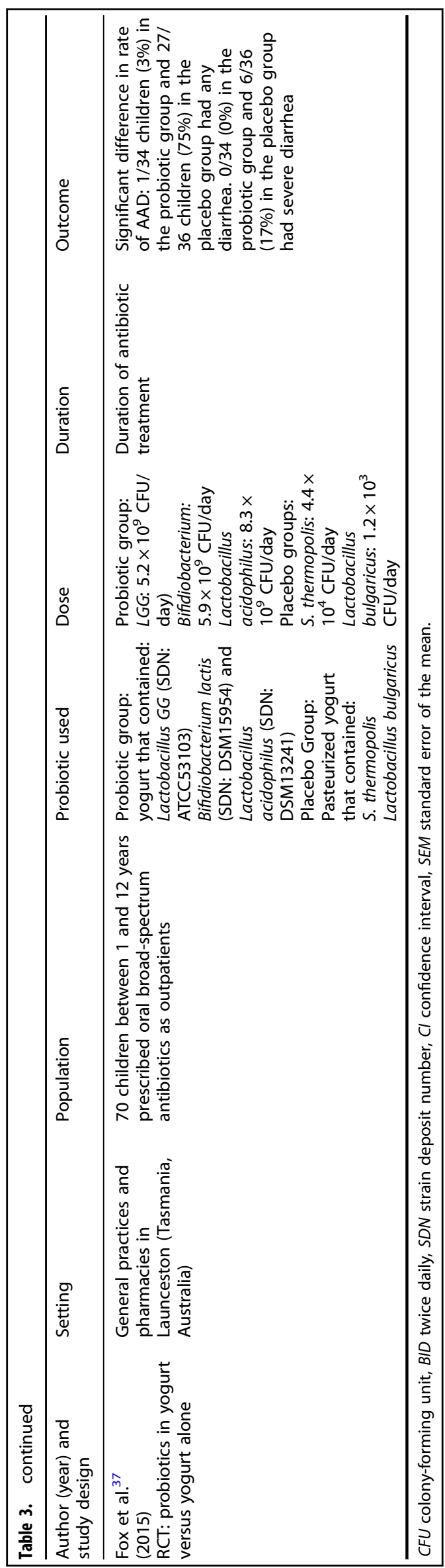

which intestinal dysbiosis leads to diarrhea is thought to be through altered digestion. Bacteria that are normally found within the colonic microbiome (i.e., Clostridium) ferment carbohydrates that were not absorbed in the small intestine into short-chain fatty acids once they pass into the colon. In the absence of these bacteria, which can occur following exposure to broad-spectrum antibiotics, the residual carbohydrates are not fermented and cause an osmotic diarrhea. ${ }^{61,62}$ Given the hypothesized relationship between antibiotics, dysbiosis, and $A D D$, there is much interest in the utility of probiotics to prevent or treat AAD.

We found studies that suggest that some specific probiotic strains may have some utility in preventing $A A D$, although in most children there are few significant consequences to AAD. Therefore, while probiotics may have some utility in preventing $A A D$, this may only have a limited clinical impact. Similarly, a 2019 Cochrane review on this topic found moderate evidence for the use of highdose probiotics ( $\geqq 5$ billion CFUs/day) to prevent AAD, with the majority of the evidence supporting the use of either $L$. rhamnosus GG or S. boulardii. ${ }^{38}$

However, in the 2020 American Gastroenterological Association's clinical practice guidelines on the use of probiotics, they do not comment on the use of probiotics for prevention of non- $C$. difficile colitis. The authors do, however, offer a conditional recommendation based on low-quality evidence to use probiotics to prevent $C$. difficile colitis in children on antibiotics. ${ }^{63}$

Much like the prior two conditions discussed, the significant heterogeneity in the literature on the use of probiotics in AAD is problematic when making recommendations regarding their use. There are also additional clinical considerations regarding the clinical use of probiotics for AAD. First, most patients may need to pay for probiotics "out of pocket," which may present a financial burden. This concern is an important balancing measure to consider when deciding to initiate probiotics for the prevention of $A A D$, especially given that not every child given antibiotics will develop AAD. Overall, the number needed to treat to prevent AAD is approximately $11 .^{64,65}$ While this is a reasonable number needed to treat, it is an important consideration when weighing the decision to prescribe probiotics for a patient for whom the cost of probiotics is prohibitive. Additionally, while probiotics are generally safe and well tolerated, there is the possibility that the increased complexity of the medication regimen may affect patient adherence with the antibiotic therapy. Indeed, the number of doses of medications has been demonstrated to be a factor in patient non-adherence with medication regimens in patients with chronic diseases. ${ }^{66-68}$ Finally, there is little oversight of the production and quality of commercially available probiotics. This was highlighted in a study of 14 commercially available probiotics, which found that only 1 of the 14 products studied contained the exact organisms listed on the label. ${ }^{69}$

\section{SOCIETY FOR PEDIATRIC RESEARCH PERSPECTIVE}

There are many areas of future research based on the current knowledge from clinical trials in these areas. These include assessing whether the native microbiota affects the clinical efficacy of a potentially therapeutic probiotic and exploring how the route of administration of probiotics affect changes in the microbiota in sites other than the Gl tract. This issue is especially relevant for the urine microbiome and associated urological disease, as oral probiotics have an indirect path to the urine. Indeed, while some probiotics have been identified in the stool following oral administration, ${ }^{70,71}$ the same has not been seen in the urine. A recent trial of healthy premenopausal women who were given an oral combination of $L$. rhamnosus $G R-1$ and $L$. reuteri $R C-14$ for 40 days found that none of the strains within the oral probiotic were ever identified in the urine. ${ }^{72}$ This may partially explain results from 
a Cochrane review, where the authors concluded that oral probiotics did not prevent UTIs in people with neuropathic bladder, ${ }^{73}$ whereas a more recent paper found that intravesical administration of probiotics reduced urinary symptoms in people with neuropathic bladders. ${ }^{74}$ Further, Lactobacillus has been demonstrated to be effective against uropathogenic Escherichia coli in vitro, with mechanisms that require the presence of Lactobacillus within the urine. ${ }^{75}$ With many different potential probiotic interventions, a greater understanding of the effect of the route of administration and the interaction of specific strains with the microbiota may help select specific probiotic strains for clinical trials that might be more likely to be successful.

In our review, we found many small studies. There is a need to perform large, well-powered research studies to determine the utility of probiotics in these, and other, common pediatric conditions. Large pediatric studies have been conducted for some diseases, such as acute gastroenteritis. ${ }^{76,77}$ Larger samples sizes are critical to understand variation in responses to the probiotics and to identify whether there are subpopulations that may disproportionately benefit from their use. Larger sample sizes would also allow investigations into the interplay between the host microbiota and the probiotic strain and how this relates to clinical improvement. Standardization of variables, such as outcomes, use of concurrent therapies, and reporting of strainlevel data, are needed to address the significant heterogeneity that is currently present in this body of research.

The need to report strain-level data of probiotics is critical given the increasing discussion in the literature regarding the concept of strain-disease specificity. ${ }^{8,78-81}$ In our review, we found that 19 of the 22 studies (86\%) that used bacteria as probiotics included strain-specific information about the probiotic intervention. Strainlevel data information will not only allow for direct comparisons between different studies but also to ensure that results are being accurately attributed to a specific strain, enabling translation to clinical practice.

The ability to identify components of the microbiome has led to new insights regarding the pathogenesis of disease. The correlation of dysbiosis and disease states has challenged the previous framework where a single pathogen is responsible for disease to a model focused on the effect of community dynamics. Changes in the microbiome exist in terms of community shifts. While these changes may be driven by a single organism, the result is manifest at the community level. ${ }^{82}$ For example, long-term exposure to $L$. rhamnosus GG may result in a shift in several components of the microbiome with increased abundance of Prevotella, Lactococcus, and Ruminococcus and decreased the proportion of $E$. coli in the intestinal microbiota. ${ }^{83}$ The administration of a specific strain by mouth leads to a shift in the intestinal microbiome, thus partially explaining why in vitro data are not always directly translated to the clinic.

Research into the potential therapeutic effects of probiotics remains an active area of investigation. Continued investigations into this area are critical to further our understanding of the pathophysiology of disease and ultimately enable our ability to improve child health.

\section{DISCLAIMER}

This review represents the opinions and recommendations of the authors and do not represent consensus or clinical care guidelines.

\section{AUTHOR CONTRIBUTIONS}

All authors made substantial contributions to interpretation of referenced studies, made critical revisions for important intellectual content, and approved the version to be published. In addition to the above, C.S.F. wrote the first draft of the manuscript.

\section{ADDITIONAL INFORMATION}

Competing interests: M.D.C. is a member of the United States Preventive Services Task Force (USPSTF). This article does not necessarily represent the views and policies of the USPSTF. The authors do not have any conflicts of interest to disclose.

Patient consent: No patient consent was required for this work.

Publisher's note Springer Nature remains neutral with regard to jurisdictional claims in published maps and institutional affiliations.

\section{REFERENCES}

1. Gao, Z., Tseng, C.-h, Pei, Z. \& Blaser, M. J. Molecular analysis of human forearm superficial skin bacterial biota. Proc. Natl Acad. Sci. USA 104, 2927-2932 (2007).

2. Hyman, R. W. et al. Microbes on the human vaginal epithelium. Proc. Natl Acad. Sci. USA 102, 7952-7957 (2005).

3. Eckburg, P. B. et al. Diversity of the human intestinal microbial flora. Science $\mathbf{3 0 8}$, 1635-1638 (2005).

4. Turnbaugh, P. J. et al. The Human Microbiome Project. Nature 449, 804-810 (2007).

5. Hill, C et al. The International Scientific Association for Probiotics and Prebiotics consensus statement on the scope and appropriate use of the term probiotic. Nat. Rev. Gastroenterol. Hepatol. 11, 506-514 (2014).

6. Metchnikoff, E. The Prolongation of Life: Optimistic Studies (G. P. Putnam and Sons, New York, 1910).

7. Jabr, F. Probiotics are no panacea. Sci. Am. 317, 26-27 (2017).

8. McFarland, L. V., Evans, C. T. \& Goldstein, E. J. C. Strain-specificity and diseasespecificity of probiotic efficacy: a systematic review and meta-analysis. Front. Med. 5, 124 (2018).

9. Mohseni, M. J. et al. Combination of probiotics and antibiotics in the prevention of recurrent urinary tract infection in children. Iran. J. Pediatr. 23, 430-438 (2013).

10. Sadeghi-bojd, S., Naghshizadian, R., Mazaheri, M., Ghane Sharbaf, F. \& Assadi, F. Efficacy of probiotic prophylaxis after the first febrile urinary tract infection in children with normal urinary tracts. J. Pediatric Infect. Dis. Soc. 9, 305-310 (2020).

11. Kunz, B. et al. Clinical validation and guidelines for the SCORAD Index: Consensus Report of the European Task Force on Atopic Dermatitis. Dermatology 195, 10-19 (1997).

12. Han, Y. et al. A randomized trial of Lactobacillus plantarum CJLP133 for the treatment of atopic dermatitis. Pediatr. Allergy Immunol. 23, 667-673 (2012).

13. Prakoeswa, C. R. S. et al. Lactobacillus plantarum IS-10506 supplementation reduced SCORAD in children with atopic dermatitis. Benef. Microbes 8, 833-840 (2017).

14. Woo, S.-I., Kim, J.-Y., Lee, Y.-J., Kim, N.-S. \& Hahn, Y.-S. Effect of Lactobacillus sake supplementation in children with atopic eczema-dermatitis syndrome. Ann. Allergy Asthma Immunol. 104, 343-348 (2010).

15. Wang, I.-J. \& Wang, J.-Y. Children with atopic dermatitis show clinical improvement after Lactobacillus exposure. Clin. Exp. Allergy 45, 779-787 (2015).

16. Sistek, D. et al. Is the effect of probiotics on atopic dermatitis confined to food sensitized children? Clin. Exp. Allergy 36, 629-633 (2006)

17. Navarro-López, V. et al. Effect of oral administration of a mixture of probiotic strains on SCORAD Index and use of topical steroids in young patients with moderate atopic dermatitis. JAMA Dermatol. 154, 37-43 (2017).

18. Štefan, L. et al. The reliability of the Mediterranean Diet Quality Index (KIDMED) Questionnaire. Nutrients 9, 419 (2017).

19. Yeşilova, Y., Çalka, Ö., Akdeniz, N. \& Berktaş, M. Effect of probiotics on the treatment of children with atopic dermatitis. Ann. Dermatol. 24, 189-193 (2012).

20. Yang, H.-J., Min, T. K., Lee, H. W. \& Pyun, B. Y. Efficacy of probiotic therapy on atopic dermatitis in children: a randomized, double-blind, placebo-controlled trial. Allergy Asthma Immunol. Res. 6, 208 (2014).

21. Rosenfeldt, V. et al. Effect of probiotic Lactobacillus strains in children with atopic dermatitis. J. Allergy Clin. Immunol. 111, 389-395 (2003).

22. Ahn, S. H. et al. Effects of Lactobacillus pentosus in children with allergensensitized atopic dermatitis. J. Korean Med. Sci. 35, e128 (2020).

23. Huang, R. et al. Probiotics for the treatment of atopic dermatitis in children: a systematic review and meta-analysis of randomized controlled trials. Front. Cell. Infect. Microbiol. 7, 392 (2017).

24. Makrgeorgou, A. et al. Probiotics for treating eczema. Cochrane Database Syst. Rev. CD006135 (2018)

25. Kotowska, M., Albrecht, P. \& Szajewska, H. Saccharomyces boulardii in the prevention of antibiotic-associated diarrhoea in children: a randomized double-blind placebo-controlled trial. Aliment. Pharmacol. Ther. 21, 583-590 (2005). 
26. Jindal, M., Goyal, Y., Lata, S. \& Sharma, R. K. Preventive role of probiotic in antibiotic associated diarrhoea in children. Indian J. Public Health Res. Dev. 8, 66-69 (2017).

27. Shan, L.-S. et al. Prevention and treatment of diarrhoea with Saccharomyces boulardii in children with acute lower respiratory tract infections. Benef. Microbes 4, 329-334 (2013).

28. Bin, Z. et al. The efficacy of Saccharomyces boulardii CNCM I-745 in addition to standard Helicobacter pylori eradication treatment in children. Pediatr. Gastroenterol. Hepatol. Nutr. 18, 17-22 (2015).

29. Olek, A. et al. Efficacy and safety of Lactobacillus plantarum DSM 9843 (LP299V) in the prevention of antibiotic-associated gastrointestinal symptoms in childrenrandomized, double-blind, placebo-controlled study. J. Pediatr. 186, 82-86 (2017).

30. Georgieva, M. et al. Use of the probiotic Lactobacillus reuteri DSM 17938 in the prevention of antibiotic-associated infections in hospitalized BULGARIAN children: a randomized, controlled trial. J. IMAB Annu. Proc. (Sci. Pap.) 21, 895-900 (2015).

31. Kołodziej, M. \& Szajewska, H. Lactobacillus reuteri DSM 17938 in the prevention of antibiotic-associated diarrhoea in children: a randomized clinical trial. Clin. Microbiol Infect. 25, 699-704 (2019).

32. Vanderhoof, J. A. et al. Lactobacillus GG in the prevention of antibiotic-associated diarrhea in children. J. Pediatr. 135, 564-568 (1999).

33. Arvola, T. et al. Prophylactic Lactobacillus GG reduces antibiotic-associated diarrhea in children with respiratory infections: a randomized study. Pediatrics 104, e64 (1999).

34. Ruszczyński, M., Radzikowski, A. \& Szajewska, H. Clinical trial: effectiveness of Lactobacillus rhamnosus (strains E/N, Oxy and Pen) in the prevention of antibiotic-associated diarrhoea in children. Aliment. Pharmacol. Ther. 28, 154-161 (2008)

35. Szymański, H., Armańska, M., Kowalska-Duplaga, K. \& Szajewska, H. Bifidobacterium longum PL03, Lactobacillus rhamnosus KL53A, and Lactobacillus plantarum PL02 in the prevention of antibiotic-associated diarrhea in children: a randomized controlled pilot trial. Digestion 78, 13-17 (2008).

36. Basnet, S., Gauchan, E., Adhikari, S. \& Sathian, B. Probiotics in the prevention of antibiotic associated diarrhoea in a tertiary teaching hospital in Pokhara: a prospective study. J. Clin. Diagn. Res. 11, SC11-SC13 (2017)

37. Fox, M. J., Ahuja, K. D. K., Robertson, I. K., Ball, M. J. \& Eri, R. D. Can probiotic yogurt prevent diarrhoea in children on antibiotics? A double-blind, randomised, placebo-controlled study. BMJ Open 5, 2-7 (2015).

38. Guo, Q., Goldenberg, J. Z., Humphrey, C., El Dib, R. \& Johnston, B. C. Probiotics for the prevention of pediatric antibiotic-associated diarrhea. Cochrane Database Syst. Rev. 4, CD004827 (2019).

39. Szajewska, H. \& Kołodziej, M. Systematic review with meta-analysis: Saccharomyces boulardii in the prevention of antibiotic-associated diarrhoea. Aliment. Pharmacol. Ther. 42, 793-801 (2015).

40. Szajewska, H. \& Kołodziej, M. Systematic review with meta-analysis: Lactobacillus rhamnosus GG in the prevention of antibiotic-associated diarrhoea in children and adults. Aliment. Pharmacol. Ther. 42, 1149-1157 (2015).

41. Szajewska, H. et al. Probiotics for the prevention of antibiotic-associated diarrhea in children. J. Pediatr. Gastroenterol. Nutr. 62, 495-506 (2016).

42. Xu, H. B., Jiang, R. H. \& Sheng, H. B. Meta-analysis of the effects of Bifidobacterium preparations for the prevention and treatment of pediatric antibiotic-associated diarrhea in China. Complement. Ther. Med. 33, 105-113 (2017)

43. Wolfe, A. J. et al. Evidence of uncultivated bacteria in the adult female bladder. J. Clin. Microbiol. 50, 1376-1383 (2012).

44. Hilt, E. E. et al. Urine is not sterile: use of enhanced urine culture techniques to detect resident bacterial flora in the adult female bladder. J. Clin. Microbiol. 52, 871-876 (2014)

45. Gerber, D., Forster, C. S. \& Hsieh, M. The role of the genitourinary microbiome in pediatric urology: a review. Curr. Urol. Rep. 19, 13 (2018).

46. Horwitz, D. et al. Decreased microbiota diversity associated with urinary tract infection in a trial of bacterial interference. J. Infect. 71, 358-367 (2015).

47. Nienhouse, V. et al. Interplay between bladder microbiota and urinary antimicrobial peptides: mechanisms for human urinary tract infection risk and symptom severity. PLOS ONE 9, e114185 (2014).

48. Pearce, M. M. et al. The female urinary microbiome: a comparison of women with and without urgency urinary incontinence. MBio https://doi.org/10.1128/mBio.01283-14 (2014).

49. Chan, R. C., Bruce, A. W. \& Reid, G. Adherence of cervical, vaginal and distal urethral normal microbial flora to human uroepithelial cells and the inhibition of adherence of gram-negative uropathogens by competitive exclusion. J. Urol. 131, 596-601 (1984).

50. Chapman, C. M. C., Gibson, G. R. \& Rowland, I. Anaerobe effects of single- and multi-strain probiotics on biofilm formation and in vitro adhesion to bladder cells by urinary tract pathogens. Anaerobe 27, 1-6 (2014).
51. Reid, G. et al. Oral use of Lactobacillus rhamnosus GR-1 and L. fermentum RC-14 significantly alters vaginal flora: randomized, placebo-controlled trial in 64 healthy women. FEMS Immunol. Med. Microbiol. 35, 131-134 (2003).

52. Reid, G., Chan, R. C., Bruce, A. W. \& Costerton, J. W. Prevention of urinary tract infection in rats with an indigenous Lactobacillus casei strain. Infect. Immun. 49, 320-324 (1985).

53. Gerasimov, S. V. Probiotic prophylaxis in pediatric recurrent urinary tract infections. Clin. Pediatr. 43, 95-98 (2004).

54. Lee, S. Y., Lee, E., Park, Y. M. \& Hong, S. J. Microbiome in the gut-skin axis in atopic dermatitis. Allergy Asthma Immunol. Res. 10, 354-362 (2018).

55. Huang, Y. J. et al. The microbiome in allergic disease: current understanding and future opportunities-2017 PRACTALL document of the American Academy of Allergy, Asthma \& Immunology and the European Academy of Allergy and Clinical Immunology. J. Allergy Clin. Immunol. 139, 1099-1110 (2017).

56. Kwon, H.-K. et al. Generation of regulatory dendritic cells and CD4+Foxp3+ T cells by probiotics administration suppresses immune disorders. Proc. Natl Acad. Sci. USA 107, 2159-2164 (2010).

57. Song, H., Yoo, Y., Hwang, J., Na, Y.-C. \& Kim, H. S. Faecalibacterium prausnitzii subspecies-level dysbiosis in the human gut microbiome underlying atopic dermatitis. J. Allergy Clin. Immunol. 137, 852-860 (2016).

58. Zheng, $\mathrm{H}$. et al. Altered gut microbiota composition associated with eczema in infants. PLOS ONE 11, e0166026 (2016).

59. Nylund, L. et al. Microarray analysis reveals marked intestinal microbiota aberrancy in infants having eczema compared to healthy children in at-risk for atopic disease. BMC Microbiol. 13, 12 (2013).

60. Lee, M.-J. et al. Perturbations of gut microbiome genes in infants with atopic dermatitis according to feeding type. J. Allergy Clin. Immunol. 141, 1310-1319 (2018).

61. Clausen, M. R., Bonnén, H., Tvede, M. \& Mortensen, P. B. Colonic fermentation to short-chain fatty acids is decreased in antibiotic-associated diarrhea. Gastroenterology 101, 1497-1504 (1991).

62. Silverman, M. A., Konnikova, L. \& Gerber, J. S. Impact of antibiotics on necrotizing enterocolitis and antibiotic-associated diarrhea. Gastroenterol. Clin. North Am. 46, 61-76 (2017).

63. Su, G. L. et al. AGA Clinical Practice Guidelines on the role of probiotics in the management of gastrointestinal disorders. Gastroenterology 159, 697-705 (2020).

64. Hempel, S. et al. Probiotics for the prevention and treatment of antibioticassociated diarrhea: a systematic review and meta-analysis. JAMA 307, 1959-1969 (2012).

65. Goldenberg, J. Z. et al. Probiotics for the prevention of pediatric antibioticassociated diarrhea. Cochrane Database Syst. Rev. CD004827 (2015).

66. Osterberg, L. \& Blaschke, T. Adherence to medication. N. Engl. J. Med. 353 , 487-497 (2005).

67. Kini, V. \& Michael, Ho. P. Interventions to improve medication adherence: a review. JAMA J. Am. Med. Assoc. 320, 2461-2473 (2018).

68. Golin, C. E. et al. A prospective study of predictors of adherence to combination antiretroviral medication. J. Gen. Intern. Med. 17, 756-765 (2002).

69. Marcobal, A., Underwood, M. A. \& Mills, D. A. Rapid determination of the bacterial composition of commercial probiotic products by terminal restriction fragment length polymorphism analysis. J. Pediatr. Gastroenterol. Nutr. 46, 608-611 (2008).

70. Goossens, D. et al. Survival of the probiotic, L. plantarum $299 \mathrm{v}$ and its effects on the faecal bacterial flora, with and without gastric acid inhibition. Dig. Liver Dis. 37, 44-50 (2005).

71. Dommels, Y. E. M. et al. Survival of Lactobacillus reuteri DSM 17938 and Lactobacillus rhamnosus GG in the human gastrointestinal tract with daily consumption of a low-fat probiotic spread. Appl. Environ. Microbiol. 75, 6198-6204 (2009).

72. Wolff, B. J., Price, T. K., Joyce, C. J., Wolfe, A. J. \& Mueller, E. R. Oral probiotics and the female urinary microbiome: a double-blinded randomized placebo-controlled trial. Int. Urol. Nephrol. 51, 2149-2159 (2019).

73. Toh, S.-L., Boswell-Ruys, C. L., Lee, B. S. B., Simpson, J. M. \& Clezy, K. R. Probiotics for preventing urinary tract infection in people with neuropathic bladder. Cochrane Database Syst. Rev. 9, CD010723 (2017).

74. Tractenberg, R. E. et al. Effects of intravesical Lactobacillus rhamnosus GG on urinary symptom burden in people with neurogenic lower urinary tract dysfunction. $P M \& R$ https://doi.org/10.1002/pmrj.12470 (2020).

75. Cadieux, P., Burton, J., Devillard, E. \& Reid, G. Lactobacillus by-products inhibit the growth of uropathogenic E. coli. J. Physiol. 60, 13-18 (2009).

76. Schnadower, D. et al. Lactobacillus rhamnosus GG versus placebo for acute gastroenteritis in children. N. Engl. J. Med. 379, 2002-2014 (2018).

77. Freedman, S. B. et al. Multicenter trial of a combination probiotic for children with gastroenteritis. N. Engl. J. Med. 379, 2015-2026 (2018).

78. Azaïs-Braesco, V., Bresson, J. L., Guarner, F. \& Corthier, G. Not all lactic acid bacteria are probiotics,... but some are. Br. J. Nutr. 103, 1079-1081 (2010). 
79. Klaenhammer, T. R. et al. Functional genomics of probiotic Lactobacilli. J. Clin. Gastroenterol. 42(Suppl 3 Pt 2), S160-S162 (2008).

80. Coudeyras, S., Marchandin, H., Fajon, C. \& Forestier, C. Taxonomic and strainspecific identification of the probiotic strain Lactobacillus rhamnosus 35 within the Lactobacillus casei group. Appl. Environ. Microbiol. 74, 2679-2689 (2008).

81. Berger, B. et al. Similarity and differences in the Lactobacillus acidophilus group identified by polyphasic analysis and comparative genomics. J. Bacteriol. 189, 1311-1321 (2007).

82. Seki, H. et al. Prevention of antibiotic-associated diarrhea in children by Clostridium butyricum MIYAIRI. Pediatr. Int. 45, 86-90 (2003).

83. Korpela, K. et al. Lactobacillus rhamnosus GG intake modifies preschool children's intestinal microbiota, alleviates penicillin-associated changes, and reduces antibiotic use. PLoS ONE 11, e0154012 (2016).
Open Access This article is licensed under a Creative Commons Attribution 4.0 International License, which permits use, sharing, adaptation, distribution and reproduction in any medium or format, as long as you give appropriate credit to the original author(s) and the source, provide a link to the Creative Commons license, and indicate if changes were made. The images or other third party material in this article are included in the article's Creative Commons license, unless indicated otherwise in a credit line to the material. If material is not included in the article's Creative Commons license and your intended use is not permitted by statutory regulation or exceeds the permitted use, you will need to obtain permission directly from the copyright holder. To view a copy of this license, visit http://creativecommons. org/licenses/by/4.0/.

(c) The Author(s) 2020 